\title{
The direct cost of intravenous insulin infusions to the NHS in England and Wales
}

\author{
Authors: Rajesh Rajendran, ${ }^{A}$ Anne $\operatorname{Scott}^{\mathrm{B}}$ and Gerry Rayman ${ }^{\mathrm{C}}$
}

\begin{abstract}
The cost of intravenous insulin infusion to the NHS is unknown. The aim of this study was to estimate the direct cost of insulin infusions to the NHS in England and Wales in the first 24-hour period of infusion. Data from the National Inpatient Diabetes Audit 2013 in the UK were used to estimate the number of insulin infusions in use across England and Wales. Costs were calculated for six models for setting up and maintenance of insulin infusions, depending on the extent of involvement of different healthcare professionals in the UK. In this study, the direct costs of intravenous insulin infusions to the NHS in England and Wales have been estimated to vary from $£ 6.4-8.5$ million in the first 24 -hour period on infusion. More appropriate use of these infusions could result in substantial cost savings.
\end{abstract}

KEYWORDS: Insulin, glucose, inpatients, diabetes, NHS

\section{Introduction}

In 1979, Alberti and Thomas first described the use of an intravenous insulin infusion (IVII) to manage strict perioperative glycaemic control in inpatients. The regimen consisted of concurrent administration of glucose, potassium and insulin from the same bag. ${ }^{1}$ Due to lack of flexibility, it has now evolved into a regimen in which glucose and potassium are infused via a volumetric pump and insulin is infused independently via a syringe driver. ${ }^{2}$ This regimen is used widely in both medical and surgical inpatients requiring tight glycaemic control in whom subcutaneous insulin is contraindicated for, eg hyperglycaemia in inpatients who are vomiting and unable to take fluids or food orally. This has been frequently referred to as the 'sliding scale' in the UK, though variable rate intravenous insulin infusion (VRIII) is now the preferred term as 'sliding scale' is an ambiguous term; ${ }^{3}$ thus for example in the US the term 'sliding scale' refers to variable intermittent boluses of subcutaneous insulin. ${ }^{4}$ Fixed

Authors: ${ }^{\text {A }}$ linical research fellow, Diabetes Centre, The Ipswich

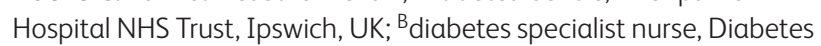
Centre, The Ipswich Hospital NHS Trust, Ipswich, UK; ' Consultant diabetologist, Diabetes Centre, The Ipswich Hospital NHS Trust, Ipswich, UK, and lead, National Inpatient Diabetes Audit, UK rate intravenous insulin infusion (FRIII) is indicated in the hospital management of diabetic emergencies, such as diabetic ketoacidosis and hyperglycaemic hyperosmolar state. ${ }^{5,6}$ The direct cost of IVIIs (FRIII and VRIII) to the NHS in the UK and the potential saving by their more appropriate use is unknown. The aim of this study was to estimate the direct cost of IVIIs to the NHS in England and Wales in the first 24-hour period of infusion.

\section{Materials and methods}

The National Inpatient Diabetes Audit (NaDIA) is conducted annually on a single day in September in a designated week, and audits the preceding seven days of inpatient diabetes care. ${ }^{7}$ Data from the NaDIA 2013 in the UK, in which more than 95\% of hospitals in England and Wales participated, was used to estimate the number of IVIIs in use across England and Wales. ${ }^{7}$ The unit cost per resource item (equipment, medication and labour) for setting up and maintenance of IVII for the first 24 hours in hospital was obtained (Table 1). Costs were calculated for six models for setting up and maintenance of IVIIs, depending on the extent of involvement of different healthcare professionals in the UK - clinical support worker, hospital ward nurse and the most junior hospital ward doctor at foundation year 1 level. The six models are presented in Table 2. In all the models, it is assumed that 24 point-of-care blood glucose tests are performed (one per hour) over a 24 -hour period and the time to perform, record and take action if necessary for one test is 5 minutes; one intravenous cannula would be sufficient for both intravenous fluids and IVII; the time to insert the cannula is 15 minutes; $2 \mathrm{~L}$ intravenous fluids would be administered over a 24-hour period via two $1 \mathrm{~L}$ bags; time to set up one bag of intravenous fluid is 10 minutes; $50 \mathrm{U}$ Actrapid insulin would be administered over a 24-hour period; and the time to set up the IVII pump is 15 minutes. Staff time devoted to point-of-care blood glucose testing, insertion of intravenous cannula, set-up and management of intravenous fluids and IVII pump was established from observation of each step in clinical practice in a single institution.

\section{Results}

As per NaDIA 2013 data, 14,198 inpatients with diabetes were audited on a single day covering their care over the previous seven days across England and Wales. Of these inpatients, 9.8\% were or had been on an $\mathrm{IVII}^{7}$ during those seven days. This 


\section{Table 1. Unit costs of healthcare (GBP).}

Resource item

Equipment and medication costs (including VAT)

1. Point-of-care bedside glucose monitoring ${ }^{a}$

Strip

Lancet

Total

2. Intravenous cannulation ${ }^{b}$

Sterile drape

Label for cannula

Swab (three pack)

$10 \mathrm{~mL}$ syringe for flush

Hypodermic $21 \mathrm{G}$ needle

$10 \mathrm{~mL} 0.9 \%$ sodium chloride solution for injection

Adhesive dressing for cannula

Octopus with two integral bionectors double lumen and two anti-reflux valves

Disposable tourniquet

Sanicloth alcohol skin wipe

Intravenous polyurethane non-ported cannula (with safety device)

Tape for removal

Sterile nitrile gloves

\section{Total}

3. Intravenous fluid therapy via pump $p^{b}$

$1 \mathrm{~L} 0.18 \%$ sodium chloride in $4 \%$ dextrose + potassium ( $27 \mathrm{mmol}$ )

Administration set for volumetric pump

$10 \mathrm{~mL}$ syringe for flush

Hypodermic $21 \mathrm{G}$ needle

$10 \mathrm{~mL} 0.9 \%$ sodium chloride solution for injection

Total

4. Intravenous insulin infusion via pump ${ }^{b}$

$50 \mathrm{~mL}$ syringe for infusion

Hypodermic $21 \mathrm{G}$ needle

$50 \mathrm{~mL} 0.9 \%$ sodium chloride solution for injection taken from $500 \mathrm{~mL}$ bag

$10 \mathrm{~mL}$ syringe for flush

Hypodermic $21 \mathrm{G}$ needle

$10 \mathrm{~mL} 0.9 \%$ sodium chloride solution for injection

Original perfusor extension set

$1 \mathrm{~mL}$ insulin syringe

Total

5. Insulin ${ }^{b}$

Actrapid $100 \mathrm{U} / \mathrm{mL}$

\section{Labour costs (staff time)c}

6. Clinical support worker

7. Hospital ward doctor (most junior doctor at foundation year-1 level)

8. Hospital ward nurse

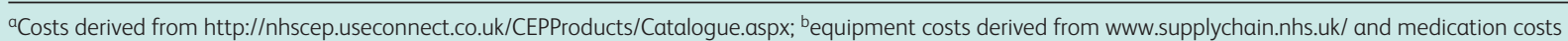
derived from www.bnf.org/bnf/index.htm, www.ppa.org.uk/edt/October_2014/mindex.htm and www.nice.org.uk/guidance/cg174/resources/intravenous-fluidtherapy-appendices2; clabour costs derived from www.pssru.ac.uk/project-pages/unit-costs/2013/.
Unit

Unit cost

Per item

Per item

0.06

$-$

0.30

Per item

0.05

Per item

0.05

Per item

0.05

Per item

0.05

Per item

0.02

Per item

0.38

Per item

0.40

Per item

2.92

Per item

Per item

0.02

Per item

0.75

Per item

0.01

Per item

-

Per item

1.25

Per item

1.70

Per item

Per item

0.02

Per item

0.38

-

Per item

0.31

Per item

0.02

Per item

0.63

Per item

0.05

Per item

Per item

0.38

Per item

0.41

Per item

0.07

-

$10 \mathrm{~mL}$ vial

Per hour

21

Perhour 25

Per hour 34 
Table 2. The six models for setting up and maintenance of intravenous insulin infusions depending on the extent of involvement of different healthcare professionals in the UK.

\begin{tabular}{llllllll} 
Healthcare professional involved & Model 1 & Model 2 & Model 3 & Model 4 & Model 5 & Model 6 \\
Point-of-care blood glucose testing & CSW & CSW & CSW & HWN & HWN & HWN \\
Intravenous cannulation & CSW & FY1 doctor & HWN & CSW & FY1 doctor & HWN \\
Intravenous fluids (setting up and maintenance) & HWN & HWN & HWN & HWN & HWN & HWN \\
Insulin infusion (setting up and maintenance) & HWN & HWN & HWN & HWN & HWN & HWN \\
\hline CSW = clinical support worker; FY1 = foundation year 1; HWN = hospital ward nurse. & & & &
\end{tabular}

equates to 72,353 inpatients on an IVII annually, assuming no seasonal variations. The estimated direct cost for the cheapest

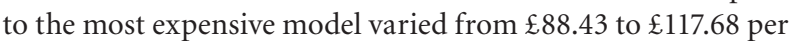
patient. The estimated annual cost in England and Wales for 72,353 inpatients varied from $\mathfrak{E} 6,398,176$ to $\mathfrak{E} 8,514,501$ in the first 24-hour period on IVII (Table 3). The majority of the costs $(>75 \%)$ were related to labour in all the models.

\section{Discussion}

This study shows that there are substantial direct costs to the NHS for just the first 24-hour period of IVII therapy. The difference in cost between the cheapest (model 1) and the most

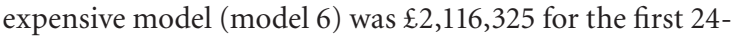
hour period of IVII in 2013. This suggests that if model 1 was universally adopted across the NHS, there would be cost savings of over $£ 2$ million. However, there are several difficulties in implementing model 1 as it is dependent on highly trained clinical support workers who can cannulate and are trained in point-of-care glucose monitoring. This is not available in many NHS trusts.

It is widely acknowledged that when used appropriately, IVII is an important mode of achieving tight glycaemic control and avoiding metabolic decompensation by accurately delivering precise amounts of insulin. ${ }^{3,8}$ However, when used incorrectly; it is associated with complications such as hyponatraemia, fluid overload, rebound ketoacidosis and hypoglycaemia, all of which can be fatal. ${ }^{3}$ Therefore it is equally important to ensure that IVIIs are not commenced inappropriately and not infused for any longer than required. The recently launched Joint British Diabetes Societies (JBDS) guidelines on the use of VRIII in medical inpatients should help in educating clinicians and training ward nurses in this regard. ${ }^{8}$ A dedicated inpatient diabetes team may also help in hospital-wide implementation of the JBDS guidelines on the use of intravenous insulin infusions. Situations where VRIII is definitely indicated are highlighted in Table $4 .{ }^{9}$
Currently, precise data on the extent of patient harm from inappropriate use of IVII is lacking. The NaDIA 2013 snapshot audit reports that $7.5 \%$ of IVIIs were used for an inappropriately long period. ${ }^{7}$ In total, there were 162 excess days on infusion in this subgroup of patients in the week of NaDIA (unpublished verified data from NaDIA 2013). Extrapolating this equates to annual costs between $£ 744,934$ (162 excess

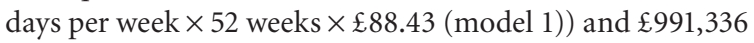
(162 excess days per week $\times 52$ weeks $\times \mathfrak{£} 117.68$ (model 6)); costs that can be potentially saved by not continuing the insulin longer than required.

One of the limitations to this study is that it only estimates direct costs and does not account for the benefits and complications (cost effectiveness) due to use of IVII in inpatients. This is because there is no data on this to estimate these costs. However, the models used in this study can be used in future studies to evaluate this if data becomes available. Another limitation of this study is the use of NaDIA data, such that the calculated costs and the potential savings are only estimates based on extrapolation of these data to a year. Since NaDIA only provides a snapshot covering a week of intravenous insulin infusion data, it could be argued that it may not accurately represent the number of infusions over a year. However, the NaDIA data do appear to be representative, as the year upon year results for the last five years are very comparable indicating the robustness of the methodology in representing IVII usage across the country. Indeed, collectively over the five years rather than one week, it could be argued that NaDIA represents five weeks of data. Apart from NaDIA there are no other reports indicative of the use of IVII across the UK.

In conclusion, this study shows that there are substantial direct costs of the IVII to the NHS for just the first 24-hour period of insulin infusion. These costs are likely to escalate steeply when infused for longer than 24 hours and costs of complications from inappropriate use of IVII are considered. Substantial cost savings could be achieved through reducing

Table 3. Estimated direct cost of intravenous insulin infusion in the first 24-hour period on infusion (GBP).

$\begin{array}{lllllll}\text { Cost } & \text { Model 1 } & \text { Model 2 } & \text { Model 3 } & \text { Model 4 } & \text { Model 5 } & \text { Model 6 } \\ \text { Estimated cost per patient: equipment and medication } & 21.35 & 21.35 & 21.35 & 21.35 & 21.35 & 21.35 \\ \text { Estimated cost per patient: labour } & 67.08 & 68.08 & 70.33 & 93.08 & 94.08 & 96.33 \\ \text { Total } & 88.43 & 89.43 & 91.68 & 114.43 & 115.43 & 117.68 \\ \text { Estimated annual cost in England and Wales (n=72,353) } & 6,398,176 & 6,470,529 & 6,633,323 & 8,279,354 & 8,351,707 & 8,514,501\end{array}$


Box 1. Indications for variable rate intravenous

\section{insulin infusion.}

Hyperglycaemia (capillary blood glucose $>10 \mathrm{mmol} / \mathrm{L}$ ) in the following situations:

> vomiting and/or intolerance of oral fluid/food (especially in type-1 diabetes after ketoacidosis has been excluded

> nil by mouth for more than one meal (eg perioperative patients, critical care unit patients)

> severe systemic illness (eg sepsis)

> special circumstances such as acute coronary syndrome, stroke and artificial feeding.

Reproduced with permission. ${ }^{9}$

the use of inappropriate insulin infusions and this may be supported by following the JBDS guidelines on the use of intravenous insulin infusions.

\section{Acknowledgements}

The authors thank Richard Driver, formulary pharmacist, and Jennis Varghese, procurement officer, for their assistance in obtaining information on the unit costs of the resource items used in this cost analysis.

\section{References}

1 Alberti KG, Thomas DJ. The management of diabetes during surgery. Br J Anaesth 1979;51:693-710.
2 Eldridge AJ, Sear JW. Peri-operative management of diabetic patients. Any changes for the better since 1985? Anaesthesia 1996;51:45-51.

3 Joint British Diabetes Societies for Inpatient Care Group. Management of adults with diabetes undergoing surgery and elective procedures: improving standards 2011. Available online at www.diabetologists-abcd.org.uk/JBDS/JBDS.htm [Accessed 12 May 2015].

4 Hirsch IB. Sliding scale insulin - time to stop sliding. JAMA 2009;301:213-4.

5 Joint British Diabetes Societies for Inpatient Care Group. The management of diabetic ketoacidosis in adults (2013). Available online at www.diabetologists-abcd.org.uk/JBDS/JBDS.htm [Accessed 12 May 2015].

6 Joint British Diabetes Societies for Inpatient Care Group. The management of the hyperosmolar hyperglycaemic state (HHS) in adults with diabetes 2012. Available online at www.diabetologists-abcd.org.uk/ JBDS/JBDS.htm [Accessed 12 May 2015].

7 Health \& Social Care Information Centre. National Diabetes Inpatient Audit. Available online at www.hscic.gov.uk/diabetesinpatientaudit [Accessed 12 May 2015].

8 Joint British Diabetes Societies for Inpatient Care Group. The use of variable rate intravenous insulin infusion (VRIII) in medical inpatients. Available online at www.diabetologists-abcd.org.uk/JBDS/JBDS.htm [Accessed 12 May 2015].

9 Rajendran R, Rayman G. Glycaemic management in patients with diabetes in hospital. Medicine 2014;42:718-22.

Address for correspondence: Dr G Rayman, Diabetes Centre, The Ipswich Hospital NHS Trust, Heath Road, Ipswich IP4 5PD, UK.

Email: gerry.rayman@ipswichhospital.nhs.uk

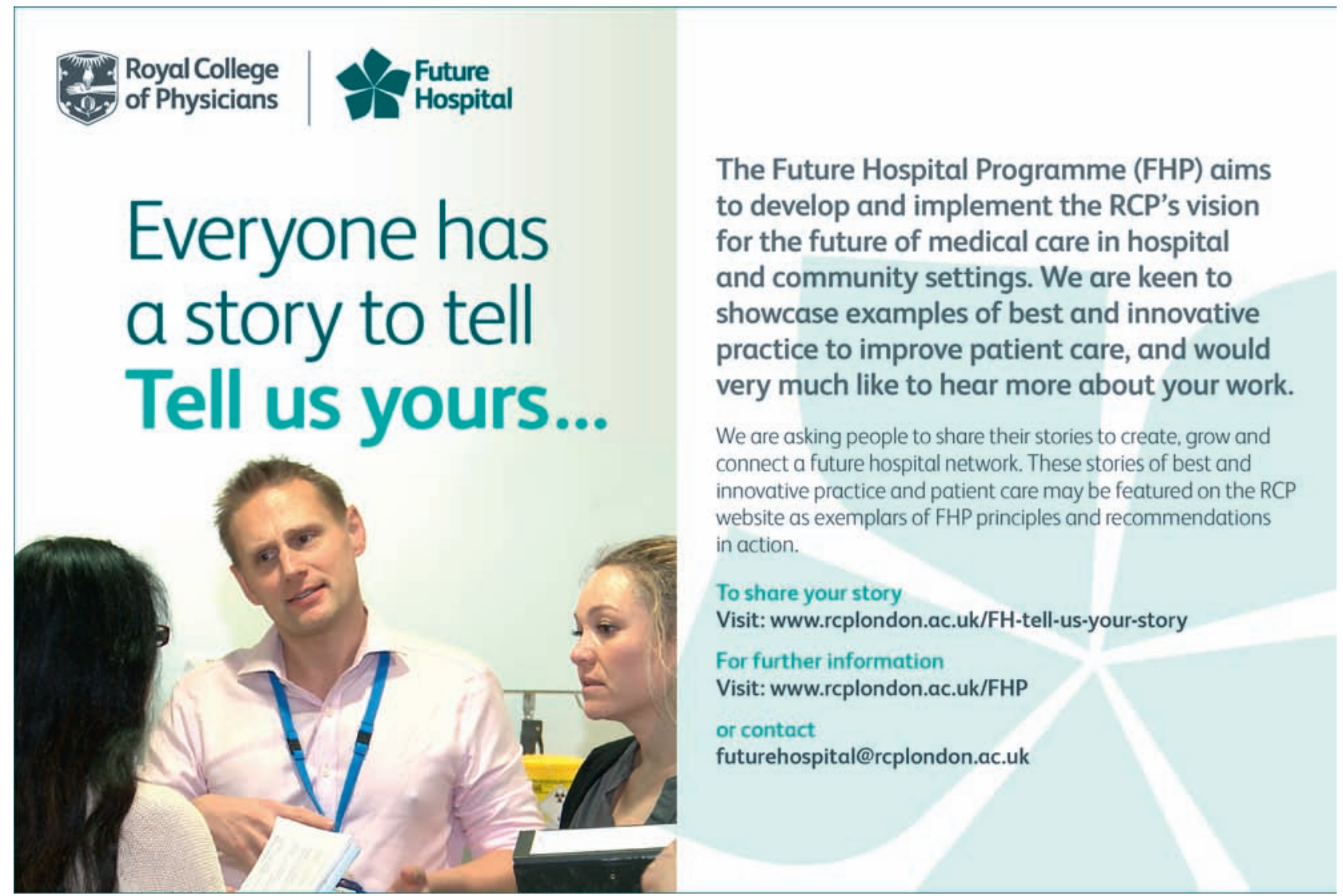

